\title{
Familial Hemophagocytic Lymphohistiocytosis (FHL), Report of two Unique Cases
}

\author{
Mishra $\mathrm{S}^{1}$, Agrawalla $\mathrm{S}^{2}$, Behura $\mathrm{SS}^{3}$, Patnaik $\mathrm{GC}^{4}$
}

\begin{abstract}
Familial hemophagocytic lymphohistiocytosis (FHLH) is a rare genetic disorder associated with early onset in life with overwhelming activation of $\mathrm{T}$ lymphocytes and macrophages invariably leading to death. We present two cases of FHLH admitted to our hospital at different points of time. First child presented with multiple episodes of GTCS and high grade fever. There was a history of sibling death before. He was having hepatosplenomegaly with leucopenia, hyper-triglyceridemia, hyper-ferritinemia and bone marrow revealed abundant hemophagocytes in smear. Second case was a 6 month male with complaint of (Generalised Tonic Clonic Seizure (GTCS) with past history of repeated attacks of acute Respiratory Infection and neuroinfection. Previous sibling died in similar presentation. He was having hepatosplenomegaly, leucopenia, hyper triglyceridemia, hyper ferritinemia with abundant hemophagocytes in bone marrow smear. Both the cases were diagnosed as FHLH and treated according to protocol.

Key words: Histiocytosis, Hemophagocytes, Pancytopenia, Stem cell
\end{abstract}

\section{Introduction}

Camilial hemophagocytic lymphohistiocytosis (FHLH) is the single - form of inherited hemophagocytic syndromes. Inheritance is autosomal recessive. The cause attributed to mutation in perforin, Munc 13-4 and syntaxin-11, all related to pathway of granule mediated cellular cytotoxicity. Hyper activation of macrophages and cytotoxic T lymphocytes (CTLs) are the primary pathogenesis. HLH is the clinical and immunological manifestation of several different disease entities, including primary or familial and secondary forms, which are associated with infections, autoimmune diseases and malignancies. FHLH is classified under class-II of histiocytic syndromes.

\section{The Case (1)}

Male child aged 3.5 months was brought with chief complaints of high grade fever for three days and multiple episodes of GTCS each lasting for 2-5 minutes with loss of consciousness. Child had no similar episode in the past. He had taken $1^{\text {st }}$ dose of DPT vaccine
'Dr. Shubhankar Mishra, MBBS, Post graduate student, Department of Paediatrics, ${ }^{2}$ Dr. Agarwalla Sunil K. MD Paediatrics, Associate Professor, Department of Paediatrics. ${ }^{3}$ Dr. Behura Sushree S. MBBS, Post graduate student, Department of Paediatrics. ${ }^{4}$ Dr. Pattanaik Gouranga C. MBBS, Post graduate student, Department of Pathology. All from the MKCG Medical College, Berhampur, Odhisa, India.

\author{
Address for correspondence: \\ Shubhankar Mishra \\ Dept. of Paediatrics, MKCG Medical college \\ Berhampur, Odisha \\ 760004 \\ E-mail:dr.subham.scb@gmail.com
}

\section{How to cite}

Mishra S, Agrawalla S, Behura SS, Patnaik GC. Familial Hemophagocytic Lymphohistiocytosis (FHL), Report of two Unique Cases. J Nepal Paediatr Soc 2015;35(3):283-286.

doi: http://dx.doi.org/10.3126/jnps.v35i3.12260

This work is licensed under a Creative Commons Attribution 3.0 License.

\section{(c) (i)}

4 days back. There was a history of similar progression and sibling death at 7 months who was diagnosed case of HLH. History of Third degree consanguineous marriage was there. There was some pallor. Heart rate $(H R)$ and respiratory rate (RR) were 146 and 38 per minute respectively. Anterior fontanelle was bulged. After 6 hours of admission his sensorium improved and all the CNS examinations were within normal range.

Hepatosplenomegaly was there with liver being $3 \mathrm{~cm}$ and spleen $4 \mathrm{~cm}$. All other systemic examinations were normal. Haemoglobin $(\mathrm{Hb})$ was $5.2 \mathrm{gm} \%$, total 
leukocyte count (TLC) was $4600 / \mathrm{mm}^{3}$, differential leukocyte count, DLC (N-46, L-53, M-01), total platelet count, $1.8 \mathrm{~L} / \mathrm{mm}^{3}$, malaria antigen kit test, negative. There was severe microcytic hypochromic anemia in peripheral smear. Serum Triglyceride (TG) was $766 \mathrm{mg} /$ $\mathrm{dl}$ and serum ferritin, 1600ng/dl. Analysis of CSF and $\mathrm{MRI}$ were normal. Bone marrow smear showed abundant hemophagocytes (Fig-1).

He was treated with IV antibiotics and IV phenytoin. Induction chemotherapy was started with Dexamethasone and Etoposide (VP-16) for 8 weeks according to $\mathrm{HLH}-94$ Protocol followed by maintenance phase with Dexamethasone, Etoposide (VP-16) and Cyclosporine $A$ and was referred to higher centre for bone marrow transplantation. But unfortunately the child succumbed there before the procedure.

\section{The Case (2)}

A six month male child was admitted with history of irregular fever for three months and repeated GTCS for three days. He was having recurrent ARI. There was history of consanguineous marriage ( $3^{\text {rd }}$ degree). Previous child died at the age of six months with similar presentation. Developmentally child was normal, exclusively breastfed and was fully immunized. On presentation child was in active seizure. His HR was 144/ min. and RR, 42/ min. He was having severe pallor. Liver was $4 \mathrm{~cm}$ and spleen $7 \mathrm{~cm}$. Anterior fontanel was normal. Hb was $4.2 \mathrm{gm} \%$, TLC-2800/ $\mathrm{mm}^{3}$ (N 8 L 87 EO M4 B1) absolute neutrophil count,224, TPC-1.2 Lac/ $\mathrm{mm}^{3}$, malaria antigen kit: (-ve), Mantoux test was negative, peripheral smear study revealed microcytic hypochromic anemia, bone marrow Smear suggested presence of abundant hemophagocytes (Fig 2).

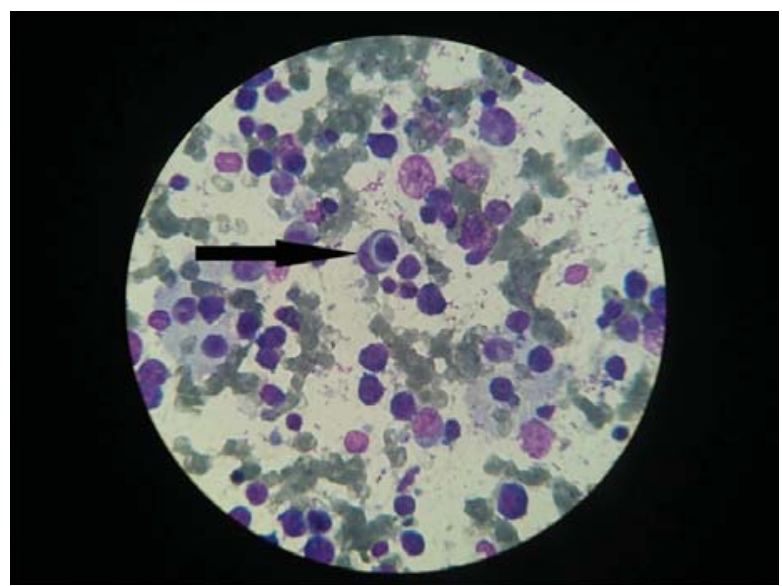

Fig 1: Arrow showing hemophagocyte in bone marrow engulfing lymphoblasts (oil immersion field 100X).

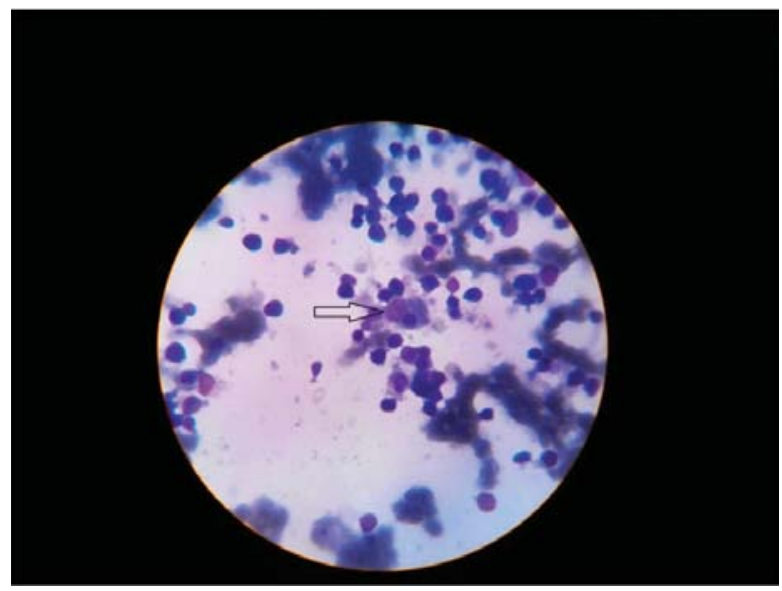

Fig 2: Arrow marked picture of hemophagocyte in bone marrow engulfing lymphoblast (oil immersion field 100X).

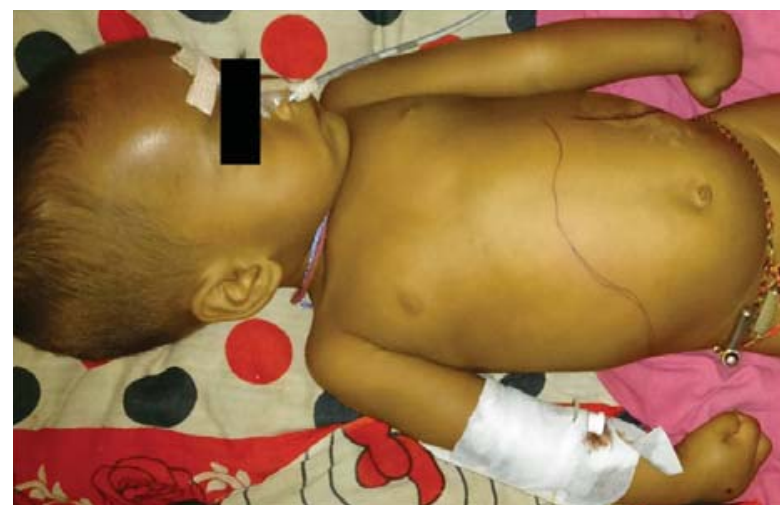

Fig 3: Child having tonic posturing with marked hepatosplenomegaly.

Serum triglyceride was $531 \mathrm{mg} / \mathrm{dl}$. Serum ferritin was $1600 \mathrm{ng} / \mathrm{dl}$. CSF analysis was normal. USG abdomen revealed hepatosplenomegaly and MRI, no abnormality. The child was treated by IV Phenytoin and IV antibiotics. Later on day 3 the child was having tonic posturing with irregular respiration (Fig 3).

He was shifted to PICU. Despite all possible intervention his clinical condition was gradually worsening. On day 4 of admission child went into respiratory depression requiring mechanical ventilation. Unfortunately he died on the evening of day 4.

\section{Discussion}

FHLH is otherwise called familial erythrophagocytic lymphohistiocytosis. The incidence is very very rare $(0 \cdot 12 / 100,000$ children born per year), with a male to female ratio of $1: 1$. The disease is classified into six different types based on genetic linkage analysis and chromosomal localization ${ }^{1}$. 
- Type 1 -unidentified gene defect located on chromosome 9

- $\quad$ Type 2-mutations in the perforin (PRF1) gene

- $\quad$ Type 3 -mutations in the Munc-13-4 (UNC13D) gene

- $\quad$ Type 4- mutations in the syntaxin 11 (STX11) gene

- $\quad$ Type 5 -mutations in the gene encoding syntaxin binding protein 2 (STXBP-2).

The incidence of the five types varies in different ethnic groups. In WHO classification FHLH is included in class II of histiocytosis ${ }^{2}$. The principal underlying defect is impaired $\mathrm{T}$ and natural killer (NK) cell cytotoxicity, but actual numbers of these effector cells are normal ${ }^{3}$. Mechanism of the defects in cytotoxicity and clinical disease is not fully understood. NK cells and CTLs play a major role in the immune response to invading pathogens, predominantly viruses. They secrete interferon-gamma, which enhances immunity, interferes with viral replication and is also a potent macrophage activator. CTLs also mediate direct killing of infected cells and antigen presenting cells (APCS) via the release of cytotoxic granules ${ }^{4}$. FHL manifest the symptoms of HLH in the first year of life with the peak age of presentation between 1 and 6 months of age $\mathrm{e}^{5}$. The most common symptoms of $\mathrm{FHL}$ are prolonged fever with or without maculopapular rash, progressive hepatosplenomegaly and cytopenias (pancytopenia or bicytopenia) ${ }^{6}$. The younger patient may present with irritability, bulging fontanelle, neck stiffness, hypotonia or hypertonia and (like our cases) ${ }^{7}$. The disease is often triggered by infections, most commonly viral, but also bacterial ${ }^{8}$. The diagnostic criteria depend upon molecular diagnosis or set of clinical picture and laboratory findings.

The diagnosis of $\mathrm{HLH}$ can be established if one of either 1 or 2 below is fulfilled ${ }^{9}$

1. A molecular diagnosis consistent with $\mathrm{HLH}$ (e.g.,PRF Mutations,SAP mutations)

2. Diagnostic criteria for HLH fulfilled (five of eight criteria below)

- Fever

- Splenomegaly

- Cytopenia [affecting at least two of the three lineages]

- Hypertriglyceridaemia and/or hypofibrinogenaemia

- Fasting triglycerides $>3.0 \mathrm{mmol} / \mathrm{l}$ (i.e. $>/=265 \mathrm{mg} /$ dl)
- $\quad$ Fibrinogen $>1.5 \mathrm{~g}(</=150 \mathrm{mg} / \mathrm{dl})$

- Haemophagocytosis in bone marrow, spleen or lymph nodes

- Low/absent natural killer cell activity

- Hyperferritinaemia (Ferritin $>500 \mathrm{ng} / \mathrm{ml}$ )

- $\quad$ High soluble interleukin 2 receptor levels (sIL-2R alpha $>/=2400 \mathrm{U} / \mathrm{ml}$ )

FHLH diagnosed by early presentation, sibling death with similar progression associated with above criteria of $\mathrm{HLH}^{2}$. In both cases more than 5 criteria were positive. Treatment primarily consists of chemotherapy and bone marrow transplantation ${ }^{9,10}$. The drugs used are etoposide (VP-16) and dexamethasone in first 8 weeks as induction phase. Maintenance phase consists of same drugs with cyclosporine A for 2-12 months. ATG had a dramatic effect in a paediatric case of refractory EBV-HLH in which the patient had been heavily treated with chemotherapy. Therapeutic plasma exchange (PE) (plasmapheresis) or exchange transfusion (ET), IVIg, and corticosteroids have been the first-choice treatment regimens most commonly employed in severe HLH cases. In particular, PE or ET is used to correct the tendency to bleed and to control hypercytokinemia.BMT done after maintenance phase. Even after BMT the prognosis of $\mathrm{FHLH}$ is bad. $60 \%$ chance of survival after $\mathrm{BMT}^{2}$.

\section{Conclusion}

$\mathrm{HLH}$ is to be suspected in any child coming with fever, hepatosplenomegaly and seizure associated with cytopenia after ruling out common infections and malignancy. Early sibling death in a case of $\mathrm{HLH}$ provides us diagnosis of FHLH. Knowledge of this entity will help the paediatricians to diagnose the disease early and provide better chance of survival.

\section{References}

1. Gholam, C., et al. "Familial haemophagocytic lymphohistiocytosis: advances in the genetic basis, diagnosis and management." Clinical \& Experimental Immunology 2011;163(3):271-83

2. StephanL. histiocytosis syndromes of childhood Nelson Textbook Of Pediatrics $19^{\text {th }}$ edition, 1773-4.

3. Jordan MB, Hildeman D, Kappler J, Marrack P. An animal model of hemophagocytic lymphohistiocytosis ( $\mathrm{HLH}): \mathrm{CD} 8+\mathrm{T}$ cells and interferon gamma are essential for the disorder. Blood 2004;1(104):735-43. 
4. Ménasché, Gael, et al. "Primary hemophagocytic syndromes point to a direct link between lymphocyte cytotoxicity and homeostasis." Immunological reviews 2005;203(1): 165-79.

5. Henter J. Hemophagocytic lymphohistiocytosis (HLH) - symptoms, signs and diagnosis of a rapidly fatal childhood disease. Histiocytosis Association of America. Available at: http:// www.histiocytesociety.org/site/c. mqISL2PIJrH/b.4442719/k.C426/ Educational_ Materialnbspnbsp.htm

6. Ishii E, Ohga S, Tanimura $M$ et al. Clinical and epidemiologic studies of familial hemophagocytic lymphohistiocytosis in Japan. Japan LCH Study Group. Med Pediatr Oncol 1998;30:276-83.
7. Filipovich, Alexandra H. "Hemophagocytic lymphohistiocytosis (HLH) and related disorders." ASH Education Program Book 2009;1:127-31.

8. Henter JI, Ehrnst A, Andersson J, Elinder G. Familial hemophagocytic lymphohistiocytosis and viral infections. Acta Paediatr 1993;82:369-72.

9. Verbsky, James W., and William J. Grossman. "Hemophagocytic lymphohistiocytosis: diagnosis, pathophysiology, treatment, and future perspectives." Annals of medicine 3006;38(1): 2031.

10. Imashuku, Shinsaku. "Advances in the management of hemophagocytic lymphohistiocytosis." International journal of hematology 2000;72(1):111. 